\title{
Comparative genome analysis of related Lymantria dispar nucleopolyhedrovirus isolates differing in virulence
}

\author{
Yu. Ilinsky ${ }^{1,2,3}$, E. Lunev², S. Toshchakov ${ }^{2,4}$, J. Podgwaite ${ }^{5}$, V. Martemyanov6,7* \\ ${ }^{1}$ Institute of Cytology and Genetics SB RAS, Novosibirsk, Russia \\ ${ }^{2}$ Immanuel Kant Baltic Federal university, Kaliningrad, Russia \\ ${ }^{3}$ Novosibirsk State University, Novosibirsk, Russia \\ ${ }^{4}$ Winogradsky Institute of Microbiology, Moscow, Russia \\ ${ }^{5}$ USDA Forest Service, Hamden, USA \\ ${ }^{6}$ Institute of systematics and ecology of animals $S B$ RAS, Novosibirsk, Russia \\ ${ }^{7}$ National Research Tomsk State University, Tomsk, Russia \\ *e-mail: martemyanov79@yahoo.com
}

Key words: nucleopolyhedrovirus, Lymantria dispar, NGS, genome, virulence

Motivation and Aim: Nucleopolyhedroviruses (NPVs, Baculoviridae) are specific viruses of insects that are used for pest control. High variation in virus virulence depends upon genetic factors [1]. Here we compare genomes of closely related genetic variants of Lymantria dispar multiple NPV (LdMNPV) that differ in virulence.

Methods and Algorithms: Five viral genotypes, differing in virulence, were isolated from the standard strain of NPV in the biopesticide "Gypchek" [2]. Complete genomes of these five genotypes were sequenced using Illumina technology by paired end sequencing of fragment genomic libraries, and assembled by SPAdes 3.9.0 [3]. Initial assemblies consisted of 1-3 scaffolds with a total assembly length of 159-174 kbp. Genome coverage was in range of $200 x-850 x$. Closing of scaffold gaps was performed by Sanger sequencing of amplicons. To determine genetic relatedness among studied LdMNPV variants a phylogenetic tree was reconstructed based on a set of core loci. Comparative analysis of candidate genes was performed to reveal genetic determinates of variation in virulence.

Results: Phylogenetic analyses indicated recent divergence of LdMNPV-studied isolates from a common ancestor. We found a number of nonsynonymous nucleotide substitutions and indels in many genes. However, we could not find a locus that could be considered as the main effector of observed variation in virulence. This fact indicated a complex genetic nature of virulence variation among closely related virus isolates.

Conclusion: Variation in virulence among related LdMNPV isolates can be explained by the complex effect of different loci.

Acknowledgements: the study was supported by RSF (grant No. 17-46-07002).

\section{References}

1. Martemyanov V.V. et al. (2017) A comparison of the adaptations of strains of Lymantria dispar multiple nucleopolyhedrovirus to hosts from spatially isolated populations. Journal of Invertebrate Pathology. 146:41-46.

2. Podgwaite J.D. et al. (2013) Potency of Nucleopolyhedrovirus Genotypes for European and Asian Gypsy Moth (Lepidoptera: Lymantriidae). Journal Entomological Science. 48(4):332-344.

3. Bankevich A. et al. (2012). SPAdes: a new genome assembly algorithm and its applications to single-cell sequencing. J Computational Biology. 19(5):455-477. 